\title{
Linear Congruence Relation and Complete Residue Systems
}

\author{
Xiquan Liang \\ Qingdao University of Science \\ and Technology \\ China
}

\author{
Li Yan \\ Qingdao University of Science \\ and Technology \\ China
}

\author{
Junjie Zhao \\ Qingdao University of Science \\ and Technology \\ China
}

\begin{abstract}
Summary. In this paper, we defined the congruence relation and proved its fundamental properties on the base of some useful theorems. Then we proved the existence of solution and the number of incongruent solution to a linear congruence and the linear congruent equation class, in particular, we proved the Chinese Remainder Theorem. Finally, we defined the complete residue system and proved its fundamental properties.
\end{abstract}

MML identifier: INT_4, version: 7.8.05 4.87.985

The papers [21], [25], [2], [20], [1], [22], [3], [24], [14], [17], [16], [23], [26], [7], [5], [27], [9], [18], [13], [19], [28], [8], [10], [4], [15], [11], [6], and [12] provide the terminology and notation for this paper.

For simplicity, we adopt the following convention: $a, b, m, x, y, i_{1}, i_{2}, i_{3}, i$ denote integers, $k, p, q, n$ denote natural numbers, $c, c_{1}, c_{2}$ denote elements of $\mathbb{N}$, and $X$ denotes a set.

Next we state the proposition

(1) For every real-membered set $X$ and for every real number $a$ holds $X \approx$ $a \oplus X$.

Let $X$ be a finite real-membered set and let $a$ be a real number. One can verify that $a \oplus X$ is finite.

One can prove the following propositions: 
(2) For every finite real-membered set $X$ and for every real number $a$ holds $\operatorname{card} X=\operatorname{card}(a \oplus X)$.

(3) For every real-membered set $X$ and for every real number $a$ such that $a \neq 0$ holds $X \approx a \circ X$.

(4) Let $X$ be a real-membered set and $a$ be a real number. Then

(i) if $a=0$ and $X$ is non empty, then $a \circ X=\{0\}$, and

(ii) if $a \circ X=\{0\}$, then $a=0$ or $X=\{0\}$.

Let $X$ be a finite real-membered set and let $a$ be a real number. One can verify that $a \circ X$ is finite.

The following propositions are true:

(5) For every finite real-membered set $X$ and for every real number $a$ such that $a \neq 0$ holds $\operatorname{card} X=\operatorname{card}(a \circ X)$.

(6) If $i \mid i_{1}$ and $i_{1} \neq 0$, then $|i| \leq\left|i_{1}\right|$.

(7) For every $i_{3}$ such that $i_{3} \neq 0$ holds $i_{1} \mid i_{2}$ iff $i_{1} \cdot i_{3} \mid i_{2} \cdot i_{3}$.

(8) For all natural numbers $a, b, m$ and for every element $n$ of $\mathbb{N}$ such that $a \bmod m=b \bmod m$ holds $a^{n} \bmod m=b^{n} \bmod m$.

(9) If $i_{1} \cdot i \equiv i_{2} \cdot i\left(\bmod i_{3}\right)$ and $i$ and $i_{3}$ are relative prime, then $i_{1} \equiv i_{2}\left(\bmod i_{3}\right)$.

(10) If $i_{1} \equiv i_{2}\left(\bmod i_{3}\right)$, then $i_{1} \cdot k \equiv i_{2} \cdot k\left(\bmod i_{3} \cdot k\right)$.

(11) If $i_{1} \equiv i_{2}(\bmod i)$, then $i_{1} \cdot i_{3} \equiv i_{2} \cdot i_{3}(\bmod i)$.

(12) For every integer $i$ holds $0=0 \bmod i$.

(13) For every $b$ such that $b>0$ and for every $a$ there exist integers $q, r$ such that $a=b \cdot q+r$ and $r \geq 0$ and $r<b$.

(14) If $i_{1} \equiv i_{2}\left(\bmod i_{3}\right)$, then $i_{1} \operatorname{gcd} i_{3}=i_{2} \operatorname{gcd} i_{3}$.

(15) If $a$ and $m$ are relative prime, then there exists an integer $x$ such that $(a \cdot x-b) \bmod m=0$.

(16) If $m>0$ and $a$ and $m$ are relative prime, then there exists a natural number $n$ such that $(a \cdot n-b) \bmod m=0$.

(17) If $m \neq 0$ and $a \operatorname{gcd} m \nmid b$, then it is not true that there exists an integer $x$ such that $(a \cdot x-b) \bmod m=0$.

(18) If $m \neq 0$ and $a \operatorname{gcd} m \mid b$, then there exists an integer $x$ such that $(a \cdot x-b) \bmod m=0$.

Let $x$ be an integer. Observe that $x^{2}$ is natural.

We now state several propositions:

(19) If $n>0$ and $p>0$, then $p \cdot q \bmod p^{n}=p \cdot\left(q \bmod p^{n-{ }^{\prime} 1}\right)$.

(20) $p \cdot q \bmod p \cdot n=p \cdot(q \bmod n)$.

(21) If $n>0$ and $p$ is prime and $p$ and $q$ are relative prime, then $p \nmid q \bmod p^{n}$.

(22) For all natural numbers $p, q, n$ such that $n>0$ holds $(p-q) \bmod n=0$ iff $p \bmod n=q \bmod n$. 
(23) For all natural numbers $a, b, m$ such that $m>0$ holds $a \bmod m=$ $b \bmod m$ iff $m \mid a-b$.

(24) If $n>0$ and $p$ is prime and $p$ and $q$ are relative prime, then it is not true that there exists an integer $x$ such that $\left(p \cdot x^{2}-q\right) \bmod p^{n}=0$.

(25) If $n>0$ and $p$ is prime and $p$ and $q$ are relative prime, then it is not true that there exists an integer $x$ such that $(p \cdot x-q) \bmod p^{n}=0$.

Let $m$ be an integer. The functor Cong $m$ yielding a binary relation on $\mathbb{Z}$ is defined as follows:

(Def. 1$) \quad\langle x, y\rangle \in \operatorname{Cong} m$ iff $x \equiv y(\bmod m)$.

Let $m$ be an integer. One can check that Cong $m$ is total.

Let $m$ be an integer. One can check that Cong $m$ is reflexive, symmetric, and transitive.

Next we state four propositions:

(26) Suppose $m \neq 0$ and $(a \cdot x-b) \bmod m=0$. Let $y$ be an integer. Then

(i) if $a$ and $m$ are relative prime and $(a \cdot y-b) \bmod m=0$, then $y \in[x]_{\operatorname{Cong} m}$, and

(ii) if $y \in[x]_{\text {Cong } m}$, then $(a \cdot y-b) \bmod m=0$.

(27) Let $a, b, m, x$ be integers. Suppose $m \neq 0$ and $a$ and $m$ are relative prime and $(a \cdot x-b) \bmod m=0$. Then there exists an integer $s$ such that $\langle x, b \cdot s\rangle \in \operatorname{Cong} m$.

(28) Let $a, m$ be elements of $\mathbb{N}$. Suppose $a \neq 0$ and $m>1$ and $a$ and $m$ are relative prime. Let $b, x$ be integers. If $(a \cdot x-b) \bmod m=0$, then $\langle x$, $\left.b \cdot a^{\text {Euler } m-^{\prime} 1}\right\rangle \in$ Cong $m$.

(29) Suppose $m \neq 0$ and $a \operatorname{gcd} m \mid b$. Then there exists a finite sequence $f_{1}$ of elements of $\mathbb{Z}$ such that len $f_{1}=a \operatorname{gcd} m$ and for every $c$ such that $c \in \operatorname{dom} f_{1}$ holds $\left(a \cdot f_{1}(c)-b\right) \bmod m=0$ and for all $c_{1}, c_{2}$ such that $c_{1} \in \operatorname{dom} f_{1}$ and $c_{2} \in \operatorname{dom} f_{1}$ and $c_{1} \neq c_{2}$ holds $f_{1}\left(c_{1}\right) \not \equiv f_{1}\left(c_{2}\right)(\bmod m)$.

We use the following convention: $f_{1}, f_{2}$ denote finite sequences of elements of $\mathbb{N}$ and $a, b, c, d, n$ denote elements of $\mathbb{N}$.

Next we state a number of propositions:

(30) For all $b, n$ such that $b \in \operatorname{dom} f_{1}$ and len $f_{1}=n+1$ holds $\left(f_{1} \frown\langle d\rangle\right)_{\mid b}=$ $\left(\left(f_{1}\right)_{\uparrow b}\right)^{\frown}\langle d\rangle$.

(31) Suppose len $f_{1} \geq 2$ and for all $b, c$ such that $b \in \operatorname{dom} f_{1}$ and $c \in \operatorname{dom} f_{1}$ and $b \neq c$ holds $\operatorname{gcd}\left(f_{1}(b), f_{1}(c)\right)=1$. Let given $b$. If $b \in \operatorname{dom} f_{1}$, then $\operatorname{gcd}\left(\prod\left(\left(f_{1}\right)_{\uparrow b}\right), f_{1}(b)\right)=1$.

(32) For every $a$ such that $a \in \operatorname{dom} f_{1}$ holds $f_{1}(a) \mid \prod f_{1}$.

(33) If $a \in \operatorname{dom} f_{1}$ and $p \mid f_{1}(a)$, then $p \mid \prod f_{1}$.

(34) If len $f_{1}=n+1$ and $a \geq 1$ and $a \leq n$, then $\left(f_{1}\right)_{\lceil a}(n)=f_{1}\left(\right.$ len $\left.f_{1}\right)$. 
(35) For all $a, b$ such that $a \in \operatorname{dom} f_{1}$ and $b \in \operatorname{dom} f_{1}$ and $a \neq b$ and len $f_{1} \geq 1$ holds $f_{1}(b) \mid \prod\left(\left(f_{1}\right)_{\uparrow a}\right)$.

(36) If for every $b$ such that $b \in \operatorname{dom} f_{1}$ holds $a \mid f_{1}(b)$, then $a \mid \sum f_{1}$.

(37) Suppose len $f_{1} \geq 2$ and for all $b, c$ such that $b \in \operatorname{dom} f_{1}$ and $c \in \operatorname{dom} f_{1}$ and $b \neq c$ holds $\operatorname{gcd}\left(f_{1}(b), f_{1}(c)\right)=1$ and for every $b$ such that $b \in \operatorname{dom} f_{1}$ holds $f_{1}(b) \neq 0$. Let given $f_{2}$. Suppose len $f_{2}=\operatorname{len} f_{1}$. Then there exists an integer $x$ such that for every $b$ such that $b \in \operatorname{dom} f_{1}$ holds $\left(x-f_{2}(b)\right) \bmod$ $f_{1}(b)=0$.

(38) If for all $b, c$ such that $b \in \operatorname{dom} f_{1}$ and $c \in \operatorname{dom} f_{1}$ and $b \neq c$ holds $\operatorname{gcd}\left(f_{1}(b), f_{1}(c)\right)=1$ and for every $b$ such that $b \in \operatorname{dom} f_{1}$ holds $f_{1}(b) \mid a$, then $\prod f_{1} \mid a$.

(39) Suppose len $f_{1} \geq 2$ and for all $b, c$ such that $b \in \operatorname{dom} f_{1}$ and $c \in \operatorname{dom} f_{1}$ and $b \neq c$ holds $\operatorname{gcd}\left(f_{1}(b), f_{1}(c)\right)=1$ and for every $b$ such that $b \in \operatorname{dom} f_{1}$ holds $f_{1}(b)>0$. Let given $f_{2}$. Suppose len $f_{2}=\operatorname{len} f_{1}$ and for every $b$ such that $b \in \operatorname{dom} f_{1}$ holds $\left(x-f_{2}(b)\right) \bmod f_{1}(b)=0$ and $\left(y-f_{2}(b)\right) \bmod f_{1}(b)=0$. Then $x \equiv y\left(\bmod \prod f_{1}\right)$.

We follow the rules: $m_{1}, m_{2}, m_{3}, r, s, a, b, c, c_{1}, c_{2}, x$ denote integers and $n_{1}, n_{2}, n_{3}$ denote natural numbers.

The following propositions are true:

(40) Suppose $m_{1} \neq 0$ and $m_{2} \neq 0$ and $m_{1}$ and $m_{2}$ are relative prime. Then there exists an integer $r$ such that for every $x$ such that $\left(x-c_{1}\right) \bmod m_{1}=$ 0 and $\left(x-c_{2}\right) \bmod m_{2}=0$ holds $x \equiv c_{1}+m_{1} \cdot r\left(\bmod m_{1} \cdot m_{2}\right)$ and $\left(m_{1} \cdot r-\left(c_{2}-c_{1}\right)\right) \bmod m_{2}=0$.

(41) If $m_{1} \neq 0$ and $m_{2} \neq 0$ and $m_{1} \operatorname{gcd} m_{2} \nmid c_{1}-c_{2}$, then it is not true that there exists $x$ such that $\left(x-c_{1}\right) \bmod m_{1}=0$ and $\left(x-c_{2}\right) \bmod m_{2}=0$.

(42) Suppose $m_{1} \neq 0$ and $m_{2} \neq 0$ and $m_{1} \operatorname{gcd} m_{2} \mid c_{2}-c_{1}$. Then there exists $r$ such that for every $x$ such that $\left(x-c_{1}\right) \bmod m_{1}=0$ and $\left(x-c_{2}\right) \bmod m_{2}=0$ holds $x \equiv c_{1}+m_{1} \cdot r\left(\bmod \operatorname{lcm}\left(m_{1}, m_{2}\right)\right)$ and $\left(\left(m_{1} \div\left(m_{1} \operatorname{gcd} m_{2}\right)\right) \cdot r-\left(\left(c_{2}-\right.\right.\right.$ $\left.\left.\left.c_{1}\right) \div\left(m_{1} \operatorname{gcd} m_{2}\right)\right)\right) \bmod \left(m_{2} \div\left(m_{1} \operatorname{gcd} m_{2}\right)\right)=0$.

(43) Suppose $m_{1} \neq 0$ and $m_{2} \neq 0$ and $a \operatorname{gcd} m_{1} \mid c_{1}$ and $b \operatorname{gcd} m_{2} \mid c_{2}$ and $m_{1}$ and $m_{2}$ are relative prime. Then there exist integers $w, r, s$ such that

(i) for every $x$ such that $\left(a \cdot x-c_{1}\right) \bmod m_{1}=0$ and $\left(b \cdot x-c_{2}\right) \bmod m_{2}=0$ holds $x \equiv r+\left(m_{1} \div\left(a \operatorname{gcd} m_{1}\right)\right) \cdot w\left(\bmod \left(m_{1} \div\left(a \operatorname{gcd} m_{1}\right)\right) \cdot\left(m_{2} \div\left(b \operatorname{gcd} m_{2}\right)\right)\right)$,

(ii) $\quad\left(\left(a \div\left(a \operatorname{gcd} m_{1}\right)\right) \cdot r-\left(c_{1} \div\left(a \operatorname{gcd} m_{1}\right)\right)\right) \bmod \left(m_{1} \div\left(a \operatorname{gcd} m_{1}\right)\right)=0$,

(iii) $\quad\left(\left(b \div\left(b \operatorname{gcd} m_{2}\right)\right) \cdot s-\left(c_{2} \div\left(b \operatorname{gcd} m_{2}\right)\right)\right) \bmod \left(m_{2} \div\left(b \operatorname{gcd} m_{2}\right)\right)=0$, and

(iv) $\quad\left(\left(m_{1} \div\left(a \operatorname{gcd} m_{1}\right)\right) \cdot w-(s-r)\right) \bmod \left(m_{2} \div\left(b \operatorname{gcd} m_{2}\right)\right)=0$.

(44) Suppose that

(i) $m_{1} \neq 0$

(ii) $m_{2} \neq 0$,

(iii) $m_{3} \neq 0$, 
(iv) $\quad m_{1}$ and $m_{2}$ are relative prime,

(v) $m_{1}$ and $m_{3}$ are relative prime, and

(vi) $\quad m_{2}$ and $m_{3}$ are relative prime.

Then there exist $r, s$ such that for every $x$ if $(x-a) \bmod m_{1}=0$ and $(x-b) \bmod m_{2}=0$ and $(x-c) \bmod m_{3}=0$, then $x \equiv a+m_{1} \cdot r+$ $m_{1} \cdot m_{2} \cdot s\left(\bmod m_{1} \cdot m_{2} \cdot m_{3}\right)$ and $\left(m_{1} \cdot r-(b-a)\right) \bmod m_{2}=0$ and $\left(m_{1} \cdot m_{2} \cdot s-\left(c-a-m_{1} \cdot r\right)\right) \bmod m_{3}=0$.

(45) Suppose $m_{1} \neq 0$ and $m_{2} \neq 0$ and $m_{3} \neq 0$ and $m_{1} \operatorname{gcd} m_{2} \nmid a-b$ or $m_{1} \operatorname{gcd} m_{3} \nmid a-c$ or $m_{2} \operatorname{gcd} m_{3} \nmid b-c$. Then it is not true that there exists $x$ such that $(x-a) \bmod m_{1}=0$ and $(x-b) \bmod m_{2}=0$ and $(x-c) \bmod m_{3}=0$.

(46) For all non zero natural numbers $n_{1}, n_{2}, n_{3}$ holds $\operatorname{lcm}\left(\operatorname{gcd}\left(n_{1}, n_{3}\right), \operatorname{gcd}\left(n_{2}, n_{3}\right)\right)=\operatorname{gcd}\left(\operatorname{lcm}\left(n_{1}, n_{2}\right), n_{3}\right)$.

(47) Let $n_{1}, n_{2}, n_{3}$ be non zero natural numbers. Suppose $\operatorname{gcd}\left(n_{1}, n_{2}\right) \mid a-b$ and $\operatorname{gcd}\left(n_{1}, n_{3}\right) \mid a-c$ and $\operatorname{gcd}\left(n_{2}, n_{3}\right) \mid b-c$. Then there exist $r, s$ such that for every $x$ if $(x-a) \bmod n_{1}=0$ and $(x-b) \bmod n_{2}=0$ and $(x-c) \bmod n_{3}=$ 0 , then $x \equiv a+n_{1} \cdot r+\operatorname{lcm}\left(n_{1}, n_{2}\right) \cdot s\left(\bmod \operatorname{lcm}\left(\operatorname{lcm}\left(n_{1}, n_{2}\right), n_{3}\right)\right)$ and $\left(\left(n_{1} \div \operatorname{gcd}\left(n_{1}, n_{2}\right)\right) \cdot r-\left((b-a) \div \operatorname{gcd}\left(n_{1}, n_{2}\right)\right)\right) \bmod \left(n_{2} \div \operatorname{gcd}\left(n_{1}, n_{2}\right)\right)=$ 0 and $\left(\left(\operatorname{lcm}\left(n_{1}, n_{2}\right) \div \operatorname{gcd}\left(\operatorname{lcm}\left(n_{1}, n_{2}\right), n_{3}\right)\right) \cdot s-\left(\left(c-\left(a+n_{1} \cdot r\right)\right) \div\right.\right.$ $\left.\left.\operatorname{gcd}\left(\operatorname{lcm}\left(n_{1}, n_{2}\right), n_{3}\right)\right)\right) \bmod \left(n_{3} \div \operatorname{gcd}\left(\operatorname{lcm}\left(n_{1}, n_{2}\right), n_{3}\right)\right)=0$.

In the sequel $f_{1}$ denotes a finite sequence of elements of $\mathbb{N}$ and $a, b, m$ denote elements of $\mathbb{N}$.

Let $m$ be an element of $\mathbb{N}$ and let $X$ be a set. We say that $X$ is a complete residue system modulo $m$ if and only if:

(Def. 2) There exists a finite sequence $f_{1}$ of elements of $\mathbb{Z}$ such that $X=\operatorname{rng} f_{1}$ and len $f_{1}=m$ and for every $b$ such that $b \in \operatorname{dom} f_{1}$ holds $f_{1}(b) \in$ $\left[b-{ }^{\prime} 1\right]_{\text {Cong } m}$.

One can prove the following propositions:

(48) $\{a: a<m\}$ is a complete residue system modulo $m$.

(49) Let $X$ be a finite set. Suppose $X$ is a complete residue system modulo $m$. Then card $X=m$ and for all integers $x, y$ such that $x \in X$ and $y \in X$ and $x \neq y$ holds $\langle x, y\rangle \notin$ Cong $m$.

(50) $\emptyset$ is a complete residue system modulo $m$ iff $m=0$.

(51) Let $X$ be a finite set. Suppose $\operatorname{card} X=m$. Then there exists a finite sequence $f_{1}$ such that len $f_{1}=m$ and for every $a$ such that $a \in \operatorname{dom} f_{1}$ holds $f_{1}(a) \in X$ and $f_{1}$ is one-to-one.

(52) Let $X$ be a finite subset of $\mathbb{Z}$. Suppose card $X=m$ and for all integers $x, y$ such that $x \in X$ and $y \in X$ and $x \neq y$ holds $\langle x, y\rangle \notin \operatorname{Cong} m$. Then $X$ is a complete residue system modulo $m$.

In the sequel $a$ is an integer. 
The following two propositions are true:

(53) Let $X$ be a finite subset of $\mathbb{Z}$. Suppose $X$ is a complete residue system modulo $m$. Then $a \oplus X$ is a complete residue system modulo $m$.

(54) Let $X$ be a finite subset of $\mathbb{Z}$. Suppose $a$ and $m$ are relative prime and $X$ is a complete residue system modulo $m$. Then $a \circ X$ is a complete residue system modulo $m$.

\section{REFERENCES}

[1] Grzegorz Bancerek. Arithmetic of non-negative rational numbers. To appear in Formalized Mathematics.

[2] Grzegorz Bancerek. Cardinal numbers. Formalized Mathematics, 1(2):377-382, 1990.

[3] Grzegorz Bancerek. The ordinal numbers. Formalized Mathematics, 1(1):91-96, 1990.

[4] Grzegorz Bancerek. Joining of decorated trees. Formalized Mathematics, 4(1):77-82, 1993.

[5] Grzegorz Bancerek and Krzysztof Hryniewiecki. Segments of natural numbers and finite sequences. Formalized Mathematics, 1(1):107-114, 1990.

[6] Józef Białas. Some properties of the intervals. Formalized Mathematics, 5(1):21-26, 1996.

[7] Czesław Byliński. Functions and their basic properties. Formalized Mathematics, 1(1):5565, 1990.

[8] Czesław Byliński. Partial functions. Formalized Mathematics, 1(2):357-367, 1990.

[9] Czesław Byliński. The sum and product of finite sequences of real numbers. Formalized Mathematics, 1(4):661-668, 1990.

[10] Agata Darmochwał. Finite sets. Formalized Mathematics, 1(1):165-167, 1990.

[11] Noboru Endou, Katsumi Wasaki, and Yasunari Shidama. Definitions and basic properties of measurable functions. Formalized Mathematics, 9(3):495-500, 2001.

[12] Noboru Endou, Katsumi Wasaki, and Yasunari Shidama. Scalar multiple of Riemann definite integral. Formalized Mathematics, 9(1):191-196, 2001.

[13] Yoshinori Fujisawa and Yasushi Fuwa. The Euler's function. Formalized Mathematics, 6(4):549-551, 1997.

[14] Krzysztof Hryniewiecki. Basic properties of real numbers. Formalized Mathematics, $1(\mathbf{1}): 35-40,1990$.

[15] Andrzej Kondracki. The Chinese Remainder Theorem. Formalized Mathematics, 6(4):573-577, 1997.

[16] Rafał Kwiatek. Factorial and Newton coefficients. Formalized Mathematics, 1(5):887-890, 1990.

[17] Rafał Kwiatek and Grzegorz Zwara. The divisibility of integers and integer relative primes. Formalized Mathematics, 1(5):829-832, 1990.

[18] Takaya Nishiyama and Yasuho Mizuhara. Binary arithmetics. Formalized Mathematics, 4(1):83-86, 1993.

[19] Konrad Raczkowski and Paweł Sadowski. Equivalence relations and classes of abstraction. Formalized Mathematics, 1(3):441-444, 1990.

[20] Andrzej Trybulec. Subsets of complex numbers. To appear in Formalized Mathematics.

[21] Andrzej Trybulec. Tarski Grothendieck set theory. Formalized Mathematics, 1(1):9-11, 1990.

[22] Andrzej Trybulec. On the sets inhabited by numbers. Formalized Mathematics, 11(4):341$347,2003$.

[23] Andrzej Trybulec and Czesław Byliński. Some properties of real numbers. Formalized Mathematics, 1(3):445-449, 1990.

[24] Michał J. Trybulec. Integers. Formalized Mathematics, 1(3):501-505, 1990.

[25] Zinaida Trybulec. Properties of subsets. Formalized Mathematics, 1(1):67-71, 1990.

[26] Edmund Woronowicz. Relations and their basic properties. Formalized Mathematics, 1(1):73-83, 1990. 
[27] Edmund Woronowicz. Relations defined on sets. Formalized Mathematics, 1(1):181-186, 1990.

[28] Edmund Woronowicz and Anna Zalewska. Properties of binary relations. Formalized Mathematics, 1(1):85-89, 1990.

Received August 28, 2007 